\title{
Revisiting the connection between intramyocellular lipids and insulin resistance: a long and winding road
}

\author{
D. M. Muoio
}

Received: 21 March 2012 /Accepted: 23 April 2012 / Published online: 3 June 2012

(C) Springer-Verlag 2012

\begin{abstract}
In the mid-1990s, researchers began to re-examine type 2 diabetes from a more 'lipocentric' perspective; giving strong consideration to the idea that systemic lipid imbalances give rise to glucose dysregulation, rather than vice versa. At the forefront of this paradigm shift was a report by Krssak and colleagues (Diabetologia 1999; 42:113-116) showing that intramyocellular lipid content, measured via the (then) novel application of proton nuclear magnetic resonance spectroscopy, served as a robust indicator of muscle insulin sensitivity in healthy individuals. A subsequent wave of investigations produced compelling correlative evidence linking ectopic lipid deposition within skeletal myocytes to the development of obesity-associated insulin resistance. But this relationship has proven much more complex than originally imagined, and scientists today are still left wondering if and how the intramyocellular accumulation of lipid droplets has a direct bearing on insulin action. Originally viewed as a simple storage depot, the lipid droplet is now recognised as an essential and sophisticated organelle that actively participates in numerous cellular processes. This edition of 'Then and now' revisits the connection between intramuscular lipids and insulin resistance and looks to future research aimed at understanding the dynamic interplay between lipid droplet biology and metabolic health.
\end{abstract}

D. M. Muoio $(\bowtie)$

Sarah W. Stedman Nutrition and Metabolism Center,

Duke University,

Durham, NC 27710, USA

e-mail: muoio@duke.edu

D. M. Muoio

Department of Medicine, Duke University,

Durham, NC, USA

D. M. Muoio

Department of Pharmacology \& Cancer Biology, Duke University,

Durham, NC, USA
Keywords Diabetes · Fatty acids · Insulin resistance · Intramyocellular triacylglycerol $\cdot$ Lipid droplet $\cdot$ Muscle . NMR $\cdot$ Obesity
Abbreviations
IMCL Intramyocellular lipid
NMR Nuclear magnetic resonance
$\operatorname{PPAR} \alpha$ Peroxisome proliferator-activated receptor $\alpha$

\section{Then: Intramyocellular lipids emerge as a hallmark of type 2 diabetes}

Although white adipocytes are best known as the cell type that sequesters large quantities of neutral lipid, most eukaryotic cells, including skeletal myocytes, produce and store triacylglycerol in the form of lipid droplets. In the early 1970s, exercise physiologists suggested that intramyocellular lipids (IMCLs) provide a readily available fuel source for contracting muscle. This prediction was based on the finding that the lipid content of biopsied skeletal muscle declines after prolonged exercise of moderate to high intensity [1]. Subsequent investigations found that, among athletes, those who were most fit had the highest IMCL levels prior to exercise and the greatest depletion at the end of an event [2]. Increased reliance on local lipids was associated with the sparing of muscle glycogen, implying that a plentiful reservoir of IMCLs benefits endurance performance by permitting more judicious use of whole body glucose reserves.

Moving forward to the mid-1990s, interests in IMCLs shifted from their salutary contributions to physical performance to their suspected role in the aetiology of metabolic disease [3]. During this 20 year timespan the odds that an 
average middle-aged American will develop type 2 diabetes more than doubled, and the disease was gaining heightened recognition as one of the leading global health threats to modernised societies. Diabetes develops when resistance to the glucose-lowering actions of insulin combines with impaired insulin secretion, giving rise to dangerously high blood glucose levels. The worldwide surge in the incidence of diabetes was deeply rooted in an obesity epidemic linked to lifestyle habits that promote physical inactivity and overnutrition [4,5]. Approximately $80 \%$ of type 2 diabetic individuals are overweight, and longitudinal studies indicated that the prediabetic onset of insulin resistance is most often preceded by weight gain. In fact, two of the great pioneers of diabetes research, Sir Philip Randle and Dr Denis McGarry, had long proposed that the secrets to understanding this disorder lie with the demise of lipid homeostasis [5, 6]. However, despite decades of intense efforts to unravel the molecular connections between excessive adiposity, lipid dysregulation and insulin resistance, the quest to delineate the precise molecular culprit(s) responsible for this relationship had been, and still remains, a formidable biological challenge.

Providing new clues to this puzzle were emerging reports describing a negative association between the lipid content of muscle and insulin sensitivity. This relationship was evident in obese humans as well as in those with type 1 or type 2 diabetes [3-5]. Because skeletal muscle serves as the major site of insulin-stimulated glucose clearance after a meal, the idea that abnormal lipid accumulation in this specific tissue might play a key role in disrupting whole body glucose homeostasis was quickly gaining momentum. At this time, studies of muscle lipid metabolism depended on invasive measures involving tracer methodologies and multiple tissue biopsies coupled with histological and biochemical analyses. A major problem with biochemical lipid extractions is the inability of this method to distinguish intramyocellular lipid from the triacylglycerol stored within adipocytes that are interspersed between the myofibres. This was becoming an increasingly important consideration as researchers in the field began to question whether the extramyocellular lipid component was introducing scatter into the correlation between total muscle lipid content and insulin sensitivity.

In 1999, Krssak and colleagues were among the first scientists to overcome this limitation by using proton nuclear magnetic resonance (NMR) spectroscopy as a noninvasive means of monitoring IMCLs in humans [7]. This procedure detects resonances from protons associated with the methylene groups of fatty acids present in tissue triacylglycerol. Because the resonance signals produced by intramyocellular versus extramyocellular triacylglycerol differ by approximately $0.2 \mathrm{ppm}$, the method allows the separate quantification of these two anatomically and functionally discrete pools. To examine the specific association between intracellular lipid and muscle insulin sensitivity, the investigators conducted a cross-sectional analysis of IMCL content in 23 young, normal weight, non-diabetic adults. IMCL content and several other known risk factors for diabetes were analysed for potential relationships with muscle insulin sensitivity, as assessed by the hyperinsulinaemic-euglycaemic clamp technique. Linear regression analysis revealed that IMCL content was more tightly correlated to whole body and muscle insulin sensitivity than circulating lipid levels, even among healthy individuals, whereas BMI, fasting blood glucose and age were unrelated to insulin sensitivity. Moreover, in a multiple regression analysis, the correlation between IMCL content and muscle insulin sensitivity was found to be independent of BMI, fasting plasma glucose and age; indicating that this specific lipid depot is uniquely predictive of insulin action. This topic of investigation gained popularity and subsequent work applied the same or similar technologies to studies of lipid-induced organ dysfunction caused by metabolic insults such as obesity, leptin deficiency, familial lipodystrophy and polycystic ovary syndrome [8]. Collectively, these studies established a remarkably strong link between the metabolic syndrome and ectopic lipid storage in non-adipocytes, which in turn inspired a new area of biochemistry research termed 'lipotoxicity' $[4,5,8]$.

\section{Now: Intramuscular lipid droplets earn respect as sophisticated organelles}

As the case against IMCLs continued to mount, a report by Goodpaster and colleagues served as a reminder that these intramuscular lipid depots were originally examined in the context of exercise training [9]. Thus, investigators found that neutral lipid accumulation in muscles of exquisitely insulin-sensitive endurance athletes is similar to, or even greater than, that found in patients with type 2 diabetes. Likewise, both acute exercise and habitual endurance training were shown to elevate the abundance of glycerolipid biosynthetic enzymes in muscle, in accord with increased rates of IMCL synthesis [3, 10]. These phenomena, now famously known as the 'athlete's paradox', strongly suggested that neutral lipid accumulation per se is not sufficient to disrupt insulin action. The advent and widespread application of transgenic technologies permitted rigorous experimental scrutiny of this conclusion in animal models. In some cases, genetic manipulations that forced intramuscular triacylglycerol accumulation coincidently provoked a diabetic phenotype [4]. Importantly, however, this connection is not absolute and a true cause-effect relationship was contradicted by several other studies showing that IMCL accumulation induced by diverse approaches, including inhibition of lipolysis, suppression of fat oxidation and hyperactivity of the glycerolipid biosynthetic pathway, does not necessarily antagonise insulin signalling [3]. Moreover, in 
some instances these manoeuvres actually afforded protection against lipid-induced glucose intolerance [11], implying that effective sequestration of fatty acid into intramuscular lipid droplets might guard against less desirable metabolic fates.

In recent years the lipid droplet has earned new respect, not only as a safe haven for surplus energy but also as a bona fide organelle that actively participates in a diverse array of cellular functions. The phospholipid monolayer surrounding the neutral core of the droplet is decorated by a complex and dynamic network of lipid droplet coat proteins [12]. A growing number of these proteins have been shown to orchestrate droplet assembly, morphology, localisation and mobilisation. Some of these proteins are permanent residents, whereas others appear to associate with the droplet surface in a transient manner, depending on the physiological setting. Also fascinating is the recent finding that genetic ablation of a lipase required for normal mobilisation of cardiac triacylglycerol results in catastrophically low activity of peroxisome proliferator-activated receptor $\alpha$ $(\operatorname{PPAR} \alpha)[13]$, a lipid-activated nuclear receptor that promotes mitochondrial biogenesis and oxidative metabolism. Notably, normal heart function was completely rescued when these mice were treated with a pharmacological activator of a PPAR $\alpha$. These results have fuelled speculation that lipid droplets dispatch fatty acids for specific biological purposes and that IMCLs serve as an essential source of lipid signalling molecules, including those that modulate nuclear receptor function (Fig. 1).

The important questions of if and how IMCLs contribute to insulin resistance in obese individuals remain unanswered. Although the lipid droplets themselves are generally considered benign in nature it is suspected that these particles provide a steady stream of fatty acid substrate that, in turn, discourages intracellular glucose use and/or gives rise to toxic lipid intermediates that directly interfere with cellular function and insulin signalling. Specific intramyocellular lipid signalling metabolites that have been implicated as

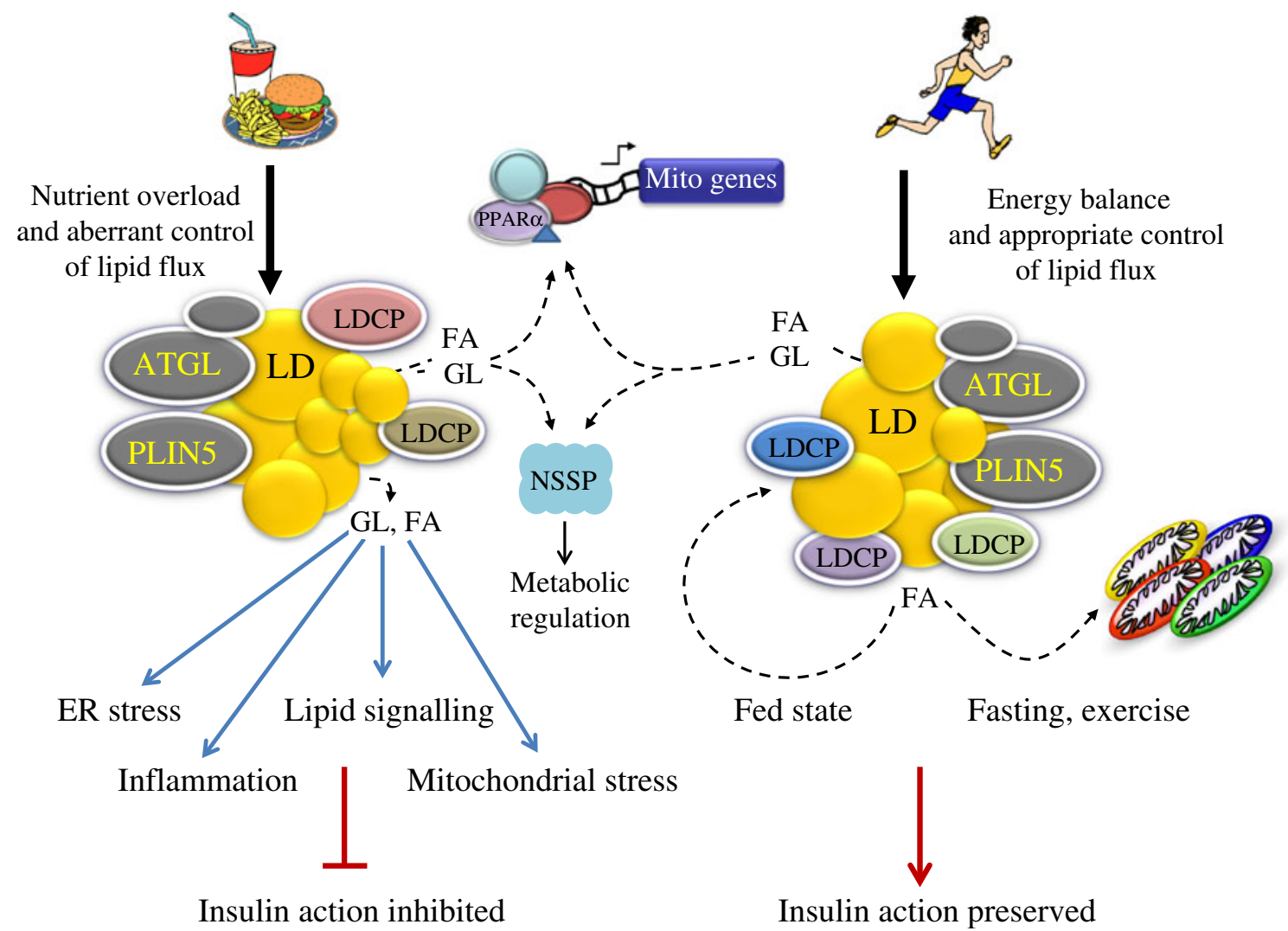

Fig. 1 Proposed interplay between IMCLs, lipid flux and insulin action. Originally viewed as an inert storage depot, intramuscular lipid droplets (LD) are now recognised as sophisticated organelles that play active roles in regulating intracellular lipid trafficking. Fatty acids (FA) and glycerolipids (GL) generated during IMCL synthesis and hydrolysis serve as fuels for neighbouring mitochondria (Mito) and as ligands for molecular targets such as PPAR $\alpha$ and other nutrientsensitive signalling proteins (NSSP). The assembly, localisation and mobilisation of LDs depend on the abundance and activities of LD coat proteins (LDCP) such as perilipin 5 (PLIN5) and adipose triacylglycerol lipase (ATGL) [12]. In the context of overnutrition, energetic surplus and LD accumulation results in aberrant control of lipid flux, thereby imposing nutrient stress on multiple organelles and NSSP. By contrast, exercise training promotes energetic balance, appropriately controlled lipid flux and enhanced insulin action, which might be attributable to a unique LD proteome resident within active muscles Dashed lines represent mobilisation and trafficking of lipid molecules 
direct mediators of insulin resistance include long-chain acylCoAs, diacylglycerol, phosphatidic acid and ceramides. Whereas the lipid signalling hypothesis has gained strong support, several other plausible theories to explain lipidinduced insulin resistance have been proposed. Prominent among these are models centred on inflammation, endoplasmic reticulum stress, mitochondrial stress and redox imbalance. Each of the aforementioned cellular insults is thought to engage stress-sensitive serine kinases that disrupt the insulin signalling cascade $[3,4]$. Although the past two decades of diabetes research have certainly advanced our understanding of the intricate metabolic and molecular events that link ectopic lipid accumulation to glucose intolerance, this topic remains a matter of debate in the field today and one that is likely to be featured in a 'Then and now' article in years to come. New insights are surfacing from studies employing transmission electron microscopy, which have produced provocative images of lipid droplets that appear to physically interact with neighbouring organelles, including mitochondria, peroxisomes, phagosomes and the endoplasmic reticulum. Consistent with this impression, recent work suggests that lipid droplets influence cellular stress responses such as autophagy, inflammation and mitochondrial production of reactive oxygen species [12]. Together, these new avenues of investigation show that IMCLs are much more sophisticated than originally imagined. The current era of proteomic and lipidomic technologies affords exciting opportunities to evaluate disease-related perturbations in the quality of IMCLs, as well as their quantity. Perhaps the reason that IMCLs do not impinge upon glucose homeostasis in physically active individuals is that the lipid droplet proteome resident within trained muscles permits enhanced control of intracellular lipid trafficking (Fig. 1). Additionally, the prevailing view in this field is that not all fat is created equal. Thus, the adverse or beneficial impact of increased IMCL content could depend on the type of fatty acids entering and exiting the droplet. These and many other intriguing possibilities are now ripe for exploration by the next generation of metabolic scientists.

Funding The author is supported by grants from the United States Public Health Service: R01 AG028930, R01 DK089312, R01 HL101189R01; and the American Diabetes Association.
Duality of interest The author declares that there is no duality of interest associated with this manuscript.

Contribution statement The author was responsible for the conception and design of the manuscript, drafting the article and approved the version to be published.

\section{References}

1. Lithell H, Orlander J, Schele R, Sjodin B, Karlsson J (1979) Changes in lipoprotein-lipase activity and lipid stores in human skeletal muscle with prolonged heavy exercise. Acta Physiol Scand 107:257-261

2. Hurley BF, Nemeth PM 3rd, Martin WH, Hagberg JM, Dalsky GP, Holloszy JO (1986) Muscle triglyceride utilization during exercise: effect of training. J Appl Physiol 60:562-567

3. Muoio DM (2010) Intramuscular triacylglycerol and insulin resistance: guilty as charged or wrongly accused? Biochim Biophys Acta 1801:281-288

4. Muoio DM, Newgard CB (2008) Mechanisms of disease: molecular and metabolic mechanisms of insulin resistance and beta-cell failure in type 2 diabetes. Nat Rev Mol Cell Biol 9:193-205

5. McGarry JD (2002) Banting lecture 2001: dysregulation of fatty acid metabolism in the etiology of type 2 diabetes. Diabetes 51:7-18

6. Randle PJ (1998) Regulatory interactions between lipids and carbohydrates: the glucose fatty acid cycle after 35 years. Diabetes Metab Rev 14:263-283 (Review)

7. Krssak M, Falk Petersen K, Dresner A et al (1999) Intramyocellular lipid concentrations are correlated with insulin sensitivity in humans: a ${ }^{1} \mathrm{H}$ NMR spectroscopy study. Diabetologia 42:113-116

8. Unger RH (2003) Minireview: weapons of lean body mass destruction: the role of ectopic lipids in the metabolic syndrome. Endocrinology 144:5159-5165

9. Goodpaster BH, He J, Watkins S, Kelley DE (2001) Skeletal muscle lipid content and insulin resistance: evidence for a paradox in endurance-trained athletes. J Clin Endocrinol Metab 86:5755-5761

10. Schenk S, Horowitz JF (2007) Acute exercise increases triglyceride synthesis in skeletal muscle and prevents fatty acid-induced insulin resistance. J Clin Invest 117:1690-1698

11. Liu L, Zhang Y, Chen N, Shi X, Tsang B, Yu YH (2007) Upregulation of myocellular DGAT1 augments triglyceride synthesis in skeletal muscle and protects against fat-induced insulin resistance. J Clin Invest 117:1679-1689

12. Fujimoto T, Parton RG (2011) Not just fat: the structure and function of the lipid droplet. Cold Spring Harb Perspect Biol 3:pii:a004838

13. Haemmerle G, Moustafa T, Woelkart G et al (2011) ATGLmediated fat catabolism regulates cardiac mitochondrial function via PPAR- $\alpha$ and PGC-1. Nat Med 17:1076-1085 